\title{
Preparation for commissioning of structural sensors of Wendelstein 7-X magnet system
}

\author{
Joris Fellinger $^{\mathrm{a}}$, Konstantin Egorov ${ }^{\mathrm{b}}$, Victor Bykov ${ }^{\mathrm{a}}$, Felix Schauer ${ }^{\mathrm{a}}$ \\ ${ }^{a}$ Max Planck Institute for Plasma Physics, EURATOM Association, Wendelsteinstr. 1, 17491 Greifswald, Germany \\ ${ }^{b}$ ITER Organization, Route de Vinon sur Verdon, 13115 St. Paul lez Durance, France
}

\begin{abstract}
The modular stellarator Wendelstein 7-X (W7-X) in Greifswald, Germany is currently under commissioning. The magnet system (MS) consists of 5 symmetric modules, each one containing two flip-symmetric half modules of 5 non-planar and 2 planar superconducting coils operated in the cryostat system (CS) at $4 \mathrm{~K}$. In order to validate the FE models that formed the basis of the design and the operational limits, the MS and CS were instrumented with more than 3000 sensors.

The paper gives an overview of the numerical tools developed to enable safe operation of the MS based on comparisons between measured sensor signals and their FE model predictions. Output extraction from the FE models was standardized for all potentially critical components and all sensor locations. A graphical user interface (GUI) was programmed to compare critical results of different simulations and models.

The GUI shows the design limit for each component. With local models, the safe design of each component was demonstrated against these design limits. The GUI also gives the degree of utilization of each component as the ratio between the calculated loads relative to the design limit. The highest degree of utilization over all components was used to define safe modes of operation and to set limits on allowed deviations of the measured signals relative to the projection before operation has to be interrupted.
\end{abstract}

Keywords: Wendelstein 7-X, magnet system, instrumentation, FE modeling, model validation, operational limits

\begin{tabular}{|c|c|c|c|}
\hline \multicolumn{4}{|c|}{ Abbreviations } \\
\hline$\overline{\mathrm{CE}}$ & Contact element & CSR & Central support ring \\
\hline $\mathrm{CS}$ & Cryostat system & DV & Design value \\
\hline EML & Electromagnetic load & FE & Finite element \\
\hline GUI & Graphical user interface & LSE & Lateral support element \\
\hline MB & Machine base & MS & Magnet system \\
\hline NPC & Non-planar coil & $\mathrm{PC}$ & Planar coil \\
\hline PSE & Planar support element & $\mathrm{SG}$ & Strain gauge \\
\hline WP & Winding pack & $3 \mathrm{D}$ & three-dimensional \\
\hline
\end{tabular}

\section{Introduction}

The modular stellarator Wendelstein $7-\mathrm{X}$ in Greifswald, Germany is currently under commissioning [1]. The magnet system (MS) consists of 5 symmetric modules each containing two flip-symmetric half modules of 5 non-planar and 2 planar superconducting coils operated in the cryostat system (CS) at $4 \mathrm{~K}$. The design of the MS is largely based on two independent FE models of a single module [2-4].

In order to validate these FE models and thus ensure safe operation, the MS and CS were instrumented with an extensive set of sensors [6-7], see table 1. The strain gauges (SG), distance sensors, displacement sensors and contact sensors are collectively referred to as machine instrumentation with 815 sensor locations.

The paper gives an overview of the development made to facilitate a fast comparison of FE models of the MS and define safe limits for measured signals. A quick interpretation of sensor signals is required to allow the operator to intervene directly if signals deviate too much from predictions in order to avoid unexpected overloading of potentially critical components. The evaluation of the sensors attached to the CS is presented in [5].

Chapter two describes the FE models of the MS. The FE models were initially used to define the location of the SGs. SGs are placed in highly stressed areas with relatively smooth gradient of stresses which are not too sensitive to any positioning inaccuracies. The overview of the sensors is given in chapter three.

Table 1: Sensors on MS and CS relevant for mechanical evaluation

\begin{tabular}{llc}
\hline Type of sensor & Location & Quantity \\
\hline Strain gauges & MS: cold part & 512 \\
& MS: warm part & 30 \\
& CS: PV & 30 \\
\hline Distance sensors & MS: flange opening & 60 \\
& MS: between coils & 70 \\
\hline Displacement sensors & MS: cryolegs & 10 \\
& CS: ports & 15 \\
\hline Contact sensors & Between MS - CS & 88 \\
\hline Vacuum pressure & CS: outer vessel & 5 \\
& CS: plasma vessel & 10 \\
\hline Temperature sensors & MS: cold part & 785 \\
& CS & 1223 \\
& Current leads & 116 \\
\hline Coil currents & & 7 \\
\hline
\end{tabular}

In chapter four, the GUI to evaluate results of the FE models is presented. This tool allows for a component wise comparison between the FE models, different electromagnetic loads (EML) and parameter settings in the simulations. In chapter five the results achieved with the GUI are shown. First, the sensitivity of sensor predictions to the mesh refinement was evaluated. Then, the maximally allowed current in individual coil group 
tests during commissioning (which is an abnormal load condition) was established without overloading any component. Finally, chapter six gives the conclusions and an outline of the planned future developments.

\section{FE models}

Two 3D FE models (one in ANSYS $\AA$ and an independent one for benchmarking in Abaqus $\left.{ }^{\circledR}\right)$ [2-4] were made of one module of the MS using the cyclic symmetry at the interface with the adjacent modules, see fig. 1. The MS exists of a central support ring (CSR) on which the non-planar coils (NPCs) and planar coils (PCs) are bolted with central support elements (CSEs). The PC casings are each made of bent inner and outer walls with two bolted cover plates using 10-12 mm pins and bolts. A CSR module is supported on the warm machine base (MB) with two cryolegs that can slide on the MB. Toroidal sliding is prevented with two tie rods. At the outboard side, the coils are welded or bolted together with lateral support elements (LSEs), and at the inboard side sliding contact pads are installed in between the NPCs. The PCs are connected to the NPCs with planar support elements (PSE) which are either sliding contact pads or bolted connections. Examples of each type of connection are shown in fig. 1.

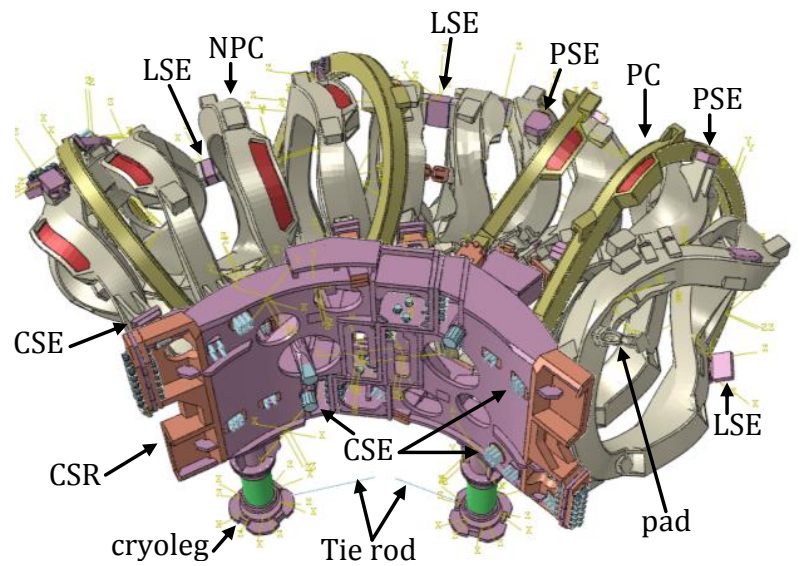

Fig. 1: FE model of $72^{\circ}$ module of MS with the coordinate systems of the sensors

The models are basically made of linear 3D solid elements and by default linear elastic behavior is applied as well as geometrical linearity. Non-linear contact behavior is included at bolted flanges, at sliding contact pads and between the superconducting winding pack (WP) and the coil cases. The model is typically loaded in four steps with pre-load of the bolts, dead weight, cool down to $4 \mathrm{~K}$ and EML. The design of the MS was determined by nine default EML configurations with $3 \mathrm{~T}$ field at the plasma axis.

For all potentially critical components, as listed in table 2, relevant output quantities through representative sections are extracted by default. These are sectional forces and moments $F_{x}, F_{y}, F_{z}$ and $M_{x}, M_{y}, M_{z}$ in solid sections, and normal force $F_{N}$, shear force $F_{s h}$, sliding $\Delta s$ and tilting $\Delta \phi$ at contacts, and normal forces, shear forces and bending moments $M_{b}$ in bolts. WP displacement vectors $u, \mathrm{WP}$ strain in current direction $\varepsilon_{\text {cur }}$, and WP rotation around the current direction $\phi_{\text {cur }}$ are also extracted.

A parameter study was performed in which uncertain model parameters like initial contact gaps, friction factors, Young's moduli and bolt pre-loads were varied. The design values (DV) of the loads were derived as the envelope of the output over all runs with consideration of a safety factor that reflects the probability of occurrence of the parameter settings. The DV were used as input in local FE models of the components to approve the structural design.

\section{Sensors}

\subsection{Locations}

First, the FE models were used to define the location of the sensors, see fig. 1. Sensors are placed in highly stressed areas which are not too sensitive to any positioning inaccuracies.

From the FE models, the predicted sensor signals are extracted in each unique sensor location in a module, see table 2 (the total number of sensors is higher as the number of locations because most sensors are repeated in several modules).

Table 2: Standard output of FE model

\begin{tabular}{|c|c|c|c|}
\hline \multicolumn{2}{|c|}{ component } & $\begin{array}{l}\text { number of } \\
\text { locations }\end{array}$ & output \\
\hline \multicolumn{2}{|l|}{ CSE } & 14 & \\
\hline \multicolumn{2}{|l|}{ LSE } & 6 & $F_{x}, F_{y}, F_{z}$ \\
\hline \multicolumn{2}{|c|}{ PSE } & 4 & $M M$ \\
\hline \multicolumn{2}{|c|}{ CSR flanges } & 3 & $M_{x}, M_{y}, M_{z}$ \\
\hline \multicolumn{2}{|c|}{ Cryolegs } & 2 & \\
\hline \multirow{2}{*}{\multicolumn{2}{|c|}{$\begin{array}{l}\text { Contact pads } \\
\text { Cryoleg bearings }\end{array}$}} & 63 & $F_{\Delta}, F_{d}, \Delta s, \Delta \phi$ \\
\hline & & 2 & $\boldsymbol{\Gamma}_{N}, \boldsymbol{\Gamma}_{s h}, \Delta S, \Delta \varphi$ \\
\hline \multicolumn{2}{|c|}{ Tie rod } & 2 & \multirow{7}{*}{$F_{N}, F_{s h}, M_{b}$} \\
\hline \multirow{5}{*}{ Bolts } & CSR flange & 42 & \\
\hline & LSE & 12 & \\
\hline & CSE & 168 & \\
\hline & PSE & 48 & \\
\hline & Cryolegs & 68 & \\
\hline \multicolumn{2}{|c|}{ PC casing bolts \& pins } & $4 \times 4 \times 96$ & \\
\hline \multicolumn{2}{|c|}{ Winding pack } & $14 \times 96$ & $u, \varepsilon_{\text {cur }}, \phi_{\text {cur }}$ \\
\hline \multirow{4}{*}{$\begin{array}{l}\text { Strain } \\
\text { gauge } \\
\text { rosettes }\end{array}$} & Coils & $14 \times 4$ & \multirow{4}{*}{$\sigma_{e q}$} \\
\hline & CSR & 28 & \\
\hline & LSE & 16 & \\
\hline & PSE & 6 & \\
\hline \multirow{3}{*}{$\begin{array}{l}\text { Strain } \\
\text { gauge } \\
\text { triplets }\end{array}$} & Bolts & 59 & \multirow{3}{*}{$\sigma_{m}, \sigma_{b}$} \\
\hline & Sleeves ${ }^{1)}$ & 3 & \\
\hline & Tie rods & 2 & \\
\hline \multicolumn{2}{|c|}{ Strain gauge cryolegs } & $2 \times 6$ & $F_{N}{ }^{2)}$ \\
\hline \multicolumn{2}{|c|}{ Coil distance } & 15 & \multirow{2}{*}{$\Delta u$} \\
\hline \multicolumn{2}{|c|}{ CSE flange opening } & 12 & \\
\hline
\end{tabular}

\subsection{Strain gauges}

All SG rosettes are compensated for temperature with a half bridge connection to a second SG on an unloaded copper plated compensation block attached to the structure in close vicinity to attain the same temperature. 
On the coils and the support structure, rosettes of 3 SGs are used in each sensor location, measuring the strain at $0^{\circ}, 45^{\circ}$ and $90^{\circ}$ inclination, thus allowing for the recalculation of the orientation- independent equivalent stress as

$$
\sigma_{e q}=\frac{E}{2} \sqrt{\left(\frac{\varepsilon_{0}+\varepsilon_{90}}{1-v}\right)^{2}+6\left(\frac{\varepsilon_{0}-\varepsilon_{45}}{1+v}\right)^{2}+6\left(\frac{\varepsilon_{90}-\varepsilon_{45}}{1+v}\right)^{2}}
$$

On the bolts and tie rods, three SG pairs (one for each pair for temperature compensation) measuring in the axial direction are equally distributed around the perimeter $\left(0^{\circ}, 120^{\circ}\right.$ and $\left.240^{\circ}\right)$. The bending and membrane stress in the bolts is recalculated as

$$
\begin{aligned}
& \sigma_{m}=\frac{E}{3}\left(\varepsilon_{0}+\varepsilon_{120}+\varepsilon_{240}\right) \\
& \sigma_{b}=\frac{2 E}{3} \sqrt{\varepsilon_{0}^{2}+\varepsilon_{120}^{2}+\varepsilon_{240}^{2}-\varepsilon_{0} \varepsilon_{120}-\varepsilon_{0} \varepsilon_{240}-\varepsilon_{120} \varepsilon_{240}}
\end{aligned}
$$

The first bolts that needed to be instrumented were already assembled and only the compressed sleeves around the bolts could be instrumented with only one SG per sleeve. So bending and membrane stress cannot be distinguished here.

On each cryoleg bearing, which can slide on the warm MB, 6 pairs of SGs measuring the strain in vertical and circumferential direction were attached. With the aid of a local FE model, a linear best fit relation between vertical load and the average strains in the vertical and circumferential direction was derived.

\subsection{Displacement sensors}

Relative displacements between coils and between bolted flanges that might open were measured with the aid of a small flexible cantilevered beam on which a SG is attached [6]. The strain is calibrated to the lateral displacement at the tip of the beam. To measure coil distances, the tip is attached to a wire that is connected to the opposite coil, and to measure flange openings, the tip is directly connected to the opposite flange.

At the feet of all ten cryolegs where the MS can slide over the MB, three potentiometers attached to the $\mathrm{MB}$ are connected to one point on the cryoleg (forming a pyramid) to measure the displacement of the cryoleg. Solving three quadratic equations, the measurements in three arbitrary directions are transformed into three orthogonal displacements. The same pyramids are installed on the outer vessel of the CS to measure the relative displacements of 15 ports.

\section{Post-processing tools}

The default output was extracted from both FE models using software dependent scripts. The output is stored in a unified Excel ${ }^{\circledR}$ template using Visual Basic $($ subroutines, resulting in one output file for each separate simulation. All output files are then imported in MATLAB ${ }^{\circledR}$ in which a graphical user interface (GUI) was programmed to allow for the final comparison of results, see fig. 2. It contains separate sheets for each type of component in which component specific output is presented. In addition, separate sheets for each type of sensor are included.

The GUI offers the possibility to compare different parameter settings, model versions and load cases for each potentially critical component. As shown in the middle picture of fig. 2, the user can select specific components or sensors, load cases and parameter settings. The selected results which are graphically presented can also be directly exported to Excel for further assessment. The GUI also offers the option to compare only the incremental results after cool down or after pre-load (pre-loaded bolts only).

The GUI further shows the DV and the degree of utilization $\mu$ of each component for a given load case as the ratio between the loads relative to the DV. For this purpose, the maximum equivalent stress was calculated in solid sections based on the DV of the six sectional force and moment components. The highest degree of utilization over all components $\mu_{\max }$ should stay below unity and thus defines the operational limit.

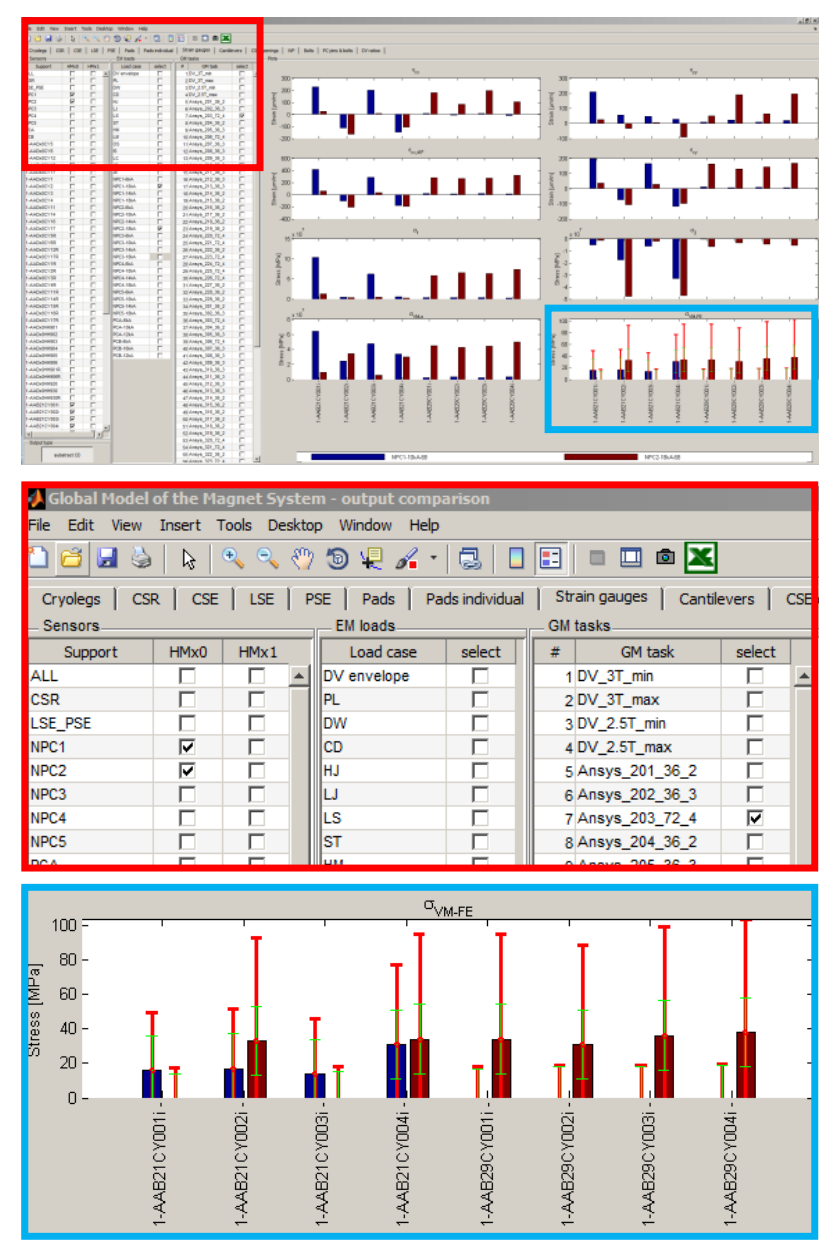

Fig. 2: Overview of GUI and zoomed views of selection area and comparison between two commissioning load simulations of equivalent stress in $8 \mathrm{SG}$ sensors distributed on NPC1 and NPC2.

It is planned to present the measured signals in the control room in a traffic light like color scheme (redyellow-green) to warn the operator in case of unexpected behavior of the MS. In fig. 2 the borders of the green and red zone are shown in the zoomed view of the SGs. Sensors will be shown in green if the measured 
signal increment $\Delta x$ does not deviate too much from the predicted increment $\Delta x_{F E}$, i.e.

$$
\min \left(\begin{array}{c}
0.8 \Delta x_{F E} \\
\Delta x_{F E}-x_{t h r}
\end{array}\right) \leq \Delta x \leq \max \left(\begin{array}{c}
1.2 \Delta x_{F E} \\
\Delta x_{F E}+x_{t h r}
\end{array}\right)
$$

The sensor dependent threshold value $x_{t h r}$ reflects the measurement uncertainty and is set to $20 \mathrm{MPa}$ for the SGs, $0.5 \mathrm{~mm}$ for the coil distance sensors and 0.1 $\mathrm{mm}$ for the flange opening sensors.

The sensors signals are presented in red if there is a risk that any potentially critical component is overloaded. As a first and simple approach, this is formulated as

$$
\mu_{\max } \Delta x_{F E}-x_{t h r} \leq \Delta x \leq \frac{\Delta x_{F E}}{\mu_{\max }}+x_{t h r}
$$

So, as an example, for a predicted SG value of 50 $\mathrm{MPa}$ and $\mu_{\max }$ equal to 0.5 , green limits are $\min (40,30)=$ $30 \mathrm{MPa}$ and $\max (60,70)=70 \mathrm{MPa}$ and red limits are $0.5 \cdot 50-20=5 \mathrm{MPa}$ and $2 \cdot 50+20=120 \mathrm{MPa}$.

\section{Results}

\subsection{Mesh refinement}

First use of the GUI was to compare SG predictions from both FE models, see fig.3. The Abaqus ${ }^{\circledR}$ mesh was refined where necessary, i.e. at the LSEs parts of the coil casings as well as at the CSR, NPC1, NPC3 and NPC4. As shown, the predictions of both models match better after refinement.

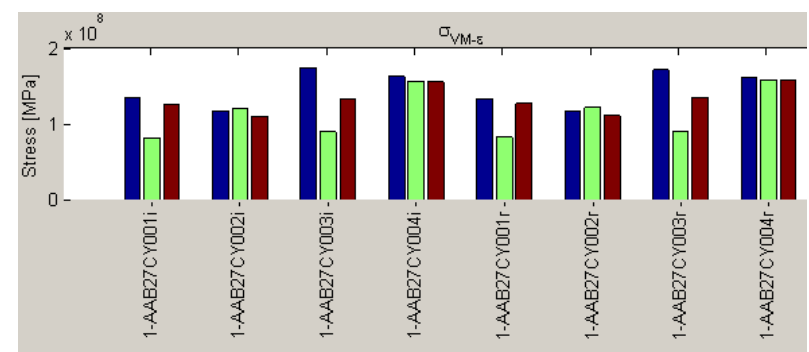

Fig. 3: Example of equivalent stress at sensor location in NPC4 for Ansys ${ }^{\circledR}$ model (blue, left) and coarse (green) and refined (red, right) mesh in Abaqus ${ }^{\circledR}$ model

\subsection{Commissioning}

During commissioning of the coils, each coil group - consisting of 10 series-connected coils of one type - is separately charged. As a result, the MS deforms significantly different than during normal operation when all coil groups are loaded simultaneously with similar current. At one hand, the currents during commissioning need to be limited to avoid overloading of any potentially critical component. At the other hand, coil tests are a great opportunity to validate the sensors, so the signals and coil currents should be significant.

\begin{tabular}{|c|c|c|c|c|c|c|c|c|}
\hline \multirow{3}{*}{\multicolumn{2}{|c|}{ component }} & \multicolumn{7}{|c|}{ relative to $3 \mathrm{~T}$, increment after $\mathrm{CD}$} \\
\hline & & \multirow{3}{*}{$\begin{array}{r}\text { NPC1 } \\
10 \mathrm{kA} \\
0.05\end{array}$} & \multirow{3}{*}{$\begin{aligned} \text { NPC2 } \\
18 \mathrm{kA} \\
0.12\end{aligned}$} & \multirow{3}{*}{$\begin{array}{l}\text { NPC3 } \\
18 \mathrm{kA} \\
0.09\end{array}$} & \multirow{3}{*}{$\begin{array}{l}\text { NPC4 } \\
18 \mathrm{kA} \\
0.09\end{array}$} & \multirow{3}{*}{$\begin{array}{l}\text { NPC5 } \\
10 \mathrm{kA} \\
0.04\end{array}$} & \multirow{3}{*}{$\begin{array}{r}\text { PCA } \\
12 \mathrm{kA} \\
0.05\end{array}$} & \multirow{3}{*}{$\begin{array}{l}\text { PCB } \\
12 \mathrm{kA} \\
0.05\end{array}$} \\
\hline & & & & & & & & \\
\hline & Cryoleg & & & & & & & \\
\hline & CSE & 0.22 & 0.43 & 0.29 & 0.29 & 0.18 & 0.23 & 0.31 \\
\hline in cross & LSE & 0.54 & 0.39 & 0.37 & 0.37 & 0.25 & 0.02 & 0.05 \\
\hline sections & PSE & 0.07 & 0.38 & 0.07 & 0.07 & 0.14 & 0.09 & 0.11 \\
\hline \multirow{2}{*}{ Pads } & force & 0.24 & 0.14 & 0.13 & 0.13 & 0.06 & 0.04 & 0.08 \\
\hline & sliding & 0.67 & 0.49 & 0.46 & 0.46 & 0.47 & 0.47 & 0.47 \\
\hline \multirow{6}{*}{$\begin{array}{l}\text { Bolt } \\
\text { loads }\end{array}$} & CSR & 0.02 & 0.01 & 0.00 & 0.00 & 0.00 & 0.01 & 0.00 \\
\hline & CSE & 0.06 & 0.12 & 0.05 & 0.05 & 0.02 & 0.02 & 0.03 \\
\hline & LSE & 0.00 & 0.01 & 0.00 & 0.00 & 0.02 & 0.00 & 0.00 \\
\hline & PSE & 0.02 & 0.05 & 0.02 & 0.02 & 0.02 & 0.03 & 0.02 \\
\hline & $\mathrm{CE}$ & 0.01 & 0.02 & 0.01 & 0.01 & 0.01 & 0.01 & 0.01 \\
\hline & cryoleg & 0.02 & 0.02 & 0.03 & 0.03 & 0.02 & 0.02 & 0.02 \\
\hline \multirow{2}{*}{$\begin{array}{c}\text { PC } \\
\text { casings }\end{array}$} & bolt & 0.01 & 0.06 & 0.02 & 0.02 & 0.01 & 0.03 & 0.04 \\
\hline & pin & 0.06 & 0.27 & 0.03 & 0.03 & 0.03 & 0.31 & 0.44 \\
\hline \multirow{2}{*}{ WP } & displ. $^{1)}$ & 0.97 & 1.11 & 0.19 & 0.19 & 0.27 & 0.33 & 0.57 \\
\hline & strain & 0.13 & 0.19 & 0.14 & 0.16 & 0.08 & 0.03 & 0.03 \\
\hline Overall & & 0.67 & 0.49 & 0.46 & 0.46 & 0.47 & 0.47 & 0.47 \\
\hline
\end{tabular}

Table 3: degree of utilization during coil testing

With the FE model of the MS, each of the 5 nonplanar coil groups was simulated with an excitation of 6 , 10,14 and $18 \mathrm{kA}$ and the 2 planar coils with 6,10 and $12 \mathrm{kA}$. Note that 18 and $12 \mathrm{kA}$ are the maximum allowed currents for the non-planar and planar coils, respectively. It was found that for all coils the maximum current could be allowed except for NPC1 and NPC5 in which $10 \mathrm{kA}$ is the limit to stay below the DV. The results are shown in table 3 . The bottom line shows the maximum degree of utilization without consideration of the WP displacement envelope because this line just indicates whether the displacement envelope used for collision checking between MS and CS is exceeded and an additional collision check between MS and CS is needed based on the deformed shape during coil testing. As the table shows, the overall degree of utilization remains below unity for each coil test.

Nevertheless, to further reduce the risk of the MS to the level that is inevitable during plasma operation, it was decided to limit the coil currents (except NPC1 and NPC5) during commissioning further to the maximum required in the first operational phase which is $12.8 \mathrm{kA}$ for the NPCs and $5.3 \mathrm{kA}$ for the PCs

\section{Conclusions and future outlook}

A GUI for FE predictions was developed which allows for a fast load and displacement comparison of all potentially critical components of the MS of W7-X and all predicted sensor signals. The GUI was already a great help in finding bugs in the models and postprocessing tools. It also yields the degrees of utilization for all load cases and all components. This feature was used to define allowed current limits during commissioning of individual coil groups, and to define limits for the sensors to warn the operator when measured signals deviate too much from the predictions. 
Another GUI for real measured data is under development. It reads in data from the central data acquisition archive, filters and transforms the raw data into meaningful values. Signals in stellarator symmetric locations are mutually compared and then compared with the FE predictions This new GUI will use the sensors limits presented here to warn the operator.

\section{Acknowledgement}

This work has been carried out within the framework of the EUROfusion Consortium and has received funding from the Euratom research and training programme 2014-2018 under grant agreement number 633053. The views and opinions expressed herein do not necessarily reflect those of the European Commission.

\section{References}

[1] H.S. Bosch et al. Transition From Construction to Operation Phase of the Wendelstein 7-X Stellarator, IEEE Transactions on Plasma Science 42 No 3. (2014) 432-438

[2] V. Bykov et al., Specific Features of Wendelstein 7-X Structural Analyses, IEEE Transaction on Plasma Science 42, No. 3, (2014) 690-697

[3] V. Bykov et al., Structural analysis of W7-X: From design to assembly and operation, Fusion Engineering and Design 86 (2011) 645-650

[4] V. Bykov et al. Numerical Modelling in the construction of Wendelstein 7-X, IEEE $25^{\text {th }}$ Symposium on Fusion Engineering (SOFE) (2013)

[5] P. van Eeten et al., Features and analyses of W7-X Cryostat system FE model, this conference

[6] Peng Chen et al. Development of a Displacement Measurement System for Wendelstein 7-X Superconducting Magnet System, IEEE Transactions on applied superconductivity, vol. 21, no. 1 (2011) 27-31

[7] Bykov et al. Wendelstein 7-x mechanical instrumentation system for commissioning and operation. Proceedings of TOFE 2014, to be published in Fusion Science and Technology 